\title{
EFFECT OF DENSITY AND DIFFERENT LEVELS OF GREEN TEA ON PRODUCTIVE PERFORMANCE, AND SOME BLOOD BIOCHEMICAL PARAMETERS OF QUAIL
}

\author{
Anwar M.Y. AL-Hamed \\ Animal Production Department, College of Agriculture and Forestry \\ University of Mosul / Iraq \\ E-mail: althuraya771@gmail.com
}

\begin{abstract}
This research was conducted at the farm of the Animal Production Department/ College of Agriculture and Forestry/University of Mosul. A total of 624 unsexed quail birds (1- 49 days) were randomly distributed in cages as follows: The $1^{\text {st }}, 2^{\text {nd }}$, $3^{\text {rd }}$, and $4^{\text {th }}$ groups were included $44 \mathrm{birds} / \mathrm{m}^{2}$ and they were fed a standard diet supplemented with $0,0.5,1$ and $1.5 \%$ green tea respectively. The $5^{\text {th }}, 6^{\text {th }}, 7^{\text {th }}$, and $8^{\text {th }}$ groups were included 52 birds $/ \mathrm{m}^{2}$ and they were fed a standard diet supplemented with $0,0.5,1$ and $1.5 \%$ green tea respectively. The $9^{\text {th }}, 10^{\text {th }}, 11^{\text {th }}$, and $12^{\text {th }}$ groups were included 60 birds $/ \mathrm{m}^{2}$ and they were fed a standard diet supplemented with 0 , $0.5,1$ and $1.5 \%$ green tea respectively. The results show a significant increase in live body weight, weight gain, improvement in feed conversion, production index and factor and albumen concentration within density $44 \mathrm{bird} / \mathrm{m}^{2}$. The same results were occurred, in addition to the decrease in cholesterol and triglycerides concentration, when adding the $1.5 \%$ green tea, while the feed consumption, mortality\%, glucose, protein, lipid profile parameters, GOT, and GPT were decreased significantly $(p \leq 0.05)$ in the same density and in the same level of adding tea that mentioned before. In the interaction between density and adding green tea, green tea traits improved most of the characters within the same density as compared with treatments without adding green tea.
\end{abstract}

Keywords: quail, density, green tea, productive performance, blood biochemical parameters.

Received: 5/12/2019 , Accepted: 9/3/2020

\section{INTRODUCTION}

In the last several years poultry production had witnessed development in meat and egg production to face the increasing demand in the global market, so the poultry producer turned to intensive quail breeding (Abou El-Ela, 2005). It has become necessary to search for new techniques to increase production, especially the optimal use of space to reduce the cost per unit to maintain the level of production when crowding consider a stressful factor that reflects on the performance of the birds (Houshmand et al., 2012). The state of health and immunity of birds is reduced in high density, which makes birds unable to get rid of heat. The use of a selective genetic improvement to raise the rate of body weight has a negative correlation to immunity response in broilers, which led breeders to use medical drugs during the breeding period to reduce the mortality and that has a negative impact reflected on the components of carcass and their effects in the health of human, that make as to 
Mesopotamia J. of Agric.

Vol. (48) No. (1) 2020
ISSN: $2224-9796$ (Online)

ISSN: 1815 - 316 X (Print)

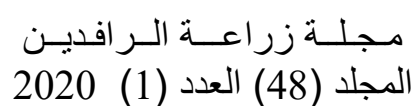

seek for medical plants that had same medical effects (Belewn et al., 2009) and as growth promoters (Çabuk et al., 2003) and antibacterial impact (Saeed and Taria, 2006) and enhance the function of liver, pancreas and small intestine, and improve the immunity (Giannenas et al., 2003). Green tea used in this research, which is one of the medical plants whose experts believe that Catchin is an active substance, which proved to be an antioxidant and has protective properties against oxidation damage (Rice et. al., 2001), Flavonoids in green tea inhibit the oxidative processes of free radicals (Jiang et al., 2003) that consider to be a carcinogen agent(Hong et al., 2009). Dipti et al., (2003) and Suad (2010) noted that the chemical matters in green tea boosted immunity against bacteria and viruses and improve the intestine environment. This called for research to find some management (nutritive) methods to reduce the effects of stress-induced density by using medicinal plants(Green Tea).

\section{MATERIALS AND METHODS}

This research included the effects of adding green tea powder at different concentrations to the quail rations in different density in production characters and blood biochemical parameters. The $1^{\text {st }}, 2^{\text {nd }}, 3^{\text {rd }}$, and $4^{\text {th }}$ groups were included 44 birds $/ \mathrm{m}^{2}$ and they were fed a standard diet supplemented with $0,0.5,1$ and $1.5 \%$ green tea respectively. The $5^{\text {th }}, 6^{\text {th }}, 7^{\text {th }}$, and $8^{\text {th }}$ groups were included: $52 \mathrm{birds} / \mathrm{m}^{2}$ and they were fed a standard diet supplemented with $0,0.5,1$ and $1.5 \%$ green tea respectively. The $9^{\text {th }}, 10^{\text {th }}, 11^{\text {th }}$, and $12^{\text {th }}$ groups were included $60 \mathrm{birds} / \mathrm{m}^{2}$ and they were fed a standard diet supplemented with $0,0.5,1$ and $1.5 \%$ green tea respectively. Chicks were reared in cages and fed on two diets formed according to the National Research Council Anonymous (1994), the starter diets: 22\% protein and $2918 \mathrm{Kcal}$ energy and grower diet: $24 \%$ protein and $3012 \mathrm{Kcal}$ energy.

Chicks were weekly weighted, quantities of feed consumed, and weights gain were measured, the conversion factor was extracted, both the production factor and index were calculated. At the end of the experiment 49 days aged, 4 birds from each replicate ( 2 males +2 females) were slaughtered and processed. The blood samples were collected in tubes without anticoagulants the serum isolated, then preserved at $\left(-20^{\circ} \mathrm{c}\right)$ till the biochemical tests were carried out, according to Friedewald et al., (1972) method, which included: estimation of glucose, cholesterol, triglyceride, and high and low density lipoprotein concentrations in serum according to the following equation :

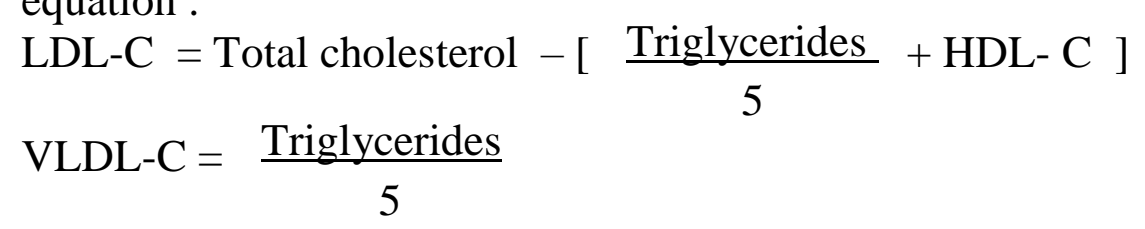

Estimation of total protein, albumin, and globulin concentration done according to Tietz (1995) method following equation:

Globulin concentration $(\mathrm{g} / 100 \mathrm{ml})=$ total protein concentration- albumin concentration. Estimation of AST and ALT concentration in serum done by using BioSystem kits.

Statistical analysis: done by completely Randomized Design (CRD) . Collected data were analyzed by Anonymous (2001), (specific groups differences were determined using Duncan's multiple range test (Steel and Torrie,1960) at $(\mathrm{p} \leq 0.05)$ significant level. and according to the model. 
Mesopotamia J. of Agric.

Vol. (48) No. (1) 2020
ISSN: $2224-9796$ (Online)

ISSN: 1815 - 316 X (Print)

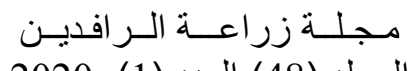

المجلد (48) العدد (1) 2020

Yij $=\mu+\mathrm{ti}+$ Eij Since: - Yij $=$ Value of observation, $\mu=$ Average of observation $=$ Treatment Effect, Eij $=$ Experimental Error.

\section{RESULTS AND DISCUSSION}

Table (1) shows a significant differences between densities in final body weight. Static analyses of data revealed, the density of $44 \mathrm{bird} / \mathrm{m}^{2}$ has higher body weight as compared with other densities, and the addition of green tea, significantly increased body weight in green tea treatments as compared with control. The interaction effects, $44 \mathrm{bird} / \mathrm{m}^{2}$ density with adding $0.5 \%$ green tea showed the best final body weight, and the lowest interaction was $44 \mathrm{bird} / \mathrm{m}^{2}$ density with $0 \%$ addition of green tea. In the same table we can notes that the treatment of $44 \mathrm{bird} / \mathrm{m} 2$ had a superior weight gain compared with $60 \mathrm{bird} / \mathrm{m} 2$ density, and there were no significant differences between the levels of added green tea, and the interaction $44 \mathrm{bird} / \mathrm{m} 2$ with $0.5 \%$ added green tea shows the best weight gain and the lowest was for the treatment $44 \mathrm{bird} / \mathrm{m} 2$ density with $0.0 \%$ added green tea, this was agreed with Feddes et al.,(2002) and Hassan (2009). From Table (1) it was clear that the $60 \mathrm{bird} / \mathrm{m} 2$ treatment consume more total feed than $52 \mathrm{bird} / \mathrm{m}^{2}$ significantly, this may be due to the high level of competition between birds, and didn't differ with $44 \mathrm{bird} / \mathrm{m}^{2}$, there was no significant effect of added green tea on feed consumption as compared to those without adding green tea. The interaction between $60 \mathrm{bird} / \mathrm{m}^{2}$ density with $0.5 \%$ added tea shows the best value of feed consumption while the lowest in 52 bird $/ \mathrm{m}^{2}$ density with 0.5 and $1.0 \%$ green tea, also from the Table (1) we note that the best feed conversion was in $44 \mathrm{bird} / \mathrm{m}^{2}$ density and in $52 \mathrm{bird} / \mathrm{m}^{2}$ density while it deteriorated through increasing the density to $60 \mathrm{bird} / \mathrm{m}^{2}$, this agreed with Siegel and Honaker (2014). Most of the feed consumed associated with uncomfortable conditions, and the best feed conversion was in the addition of green tea 1, 1.5\%. While the best interaction was in $44 \mathrm{bird} / \mathrm{m}^{2}$ density with added $0.5,1,1.5 \%$ green tea and $52 \mathrm{bird} / \mathrm{m}^{2}$ density with $1.5 \%$ green tea. It was noted that the addition of green tea had improved the conversion factor within the same density as compared to the control. This may be due to improved utilization of nutrients through improved absorption as green tea slows the passage of nutrient content due to increased feed bulk what gives more time for the action of enzymes as well as the development and growth of villi and crypts, increasing the absorption efficiency of digested substances (Latshaw, 2008 ; Crespo and Garcia, 2002).

Table (2) shows a significant increase in the mortality percent in the treatment 60 $\mathrm{bird} / \mathrm{m}^{2}$ density in $\mathrm{a} 7^{\text {th }}$ week, this results from the burden of heat stress within the herd whereas it is difficult for birds to get rid of excess heat and the emergence of cases of lack in ventilation despite the provision of good and equal ventilation, and an incensement in the level of hostility between birds and sudden death as a result of disequilibrium of internal balance processes (Metheringham and Hubrecht, 1996). In regard to green tea, the mortality\% decreased significantly in the when the green tea added with $1.5 \%$ compared to the addition of $0 \%$ green tea, because the green tea contains antioxidants that have a pharmacological effects (Suad, 2010), particularly anti-inflammatory effect that acts in the intestines and liver (Dipti et al., 2003), also influencing immunity, especially in stress conditions (Sahin et al., 2010 ; El-Deek et al., 2011). There were a significant increase in the mortality\% in the interaction 60 
Mesopotamia J. of Agric. Vol. (48) No. (1) 2020
ISSN: $2224-9796$ (Online)

ISSN: 1815 - 316 X (Print)
المجلد (48) العدة زراعـة الـر افديـن 2020

bird $/ \mathrm{m}^{2}$ density with $0 \%$ green tea while the mortality was reduced significantly in the treatment $44 \mathrm{bird} / \mathrm{m}^{2}$ with added $0.5,1.0,1.5 \%$ green tea, green tea addition may be decreased the stress of congestion by improving the immunity and the health of the birds. In regard to the effect of density in the production index, the densities 44 $\mathrm{bird} / \mathrm{m}^{2}$ and $52 \mathrm{bird} / \mathrm{m}^{2}$ were better than the treatment $60 \mathrm{bird} / \mathrm{m}^{2}$ in the $7^{\text {th }}$ week and these results were agreed with Agrawal et al., (2003) and Hassan (2009), and there was no significant difference between the treatments of added green tea in production index for birds. The best production index was $44 \mathrm{bird} / \mathrm{m}^{2}$ with $0.5 \%$ green tea and the best production factor was in interaction $44 \mathrm{bird} / \mathrm{m}^{2}$ with $0.5 \%$ green tea.

Tables (3) and (4) showed that there were no significant differences between the densities in the cholesterol $\mathrm{mg} / \mathrm{dl}$, triglycerides $\mathrm{mg} / \mathrm{dl}$, LDL $\mathrm{mg} / \mathrm{dl}, \mathrm{VLDL} \mathrm{mg} / \mathrm{dl}$, albumin g/l, GPT and GOT IU/L, concentration due to high density, the results agreed with Abdel-Azeem (2010) and Houshmand et al., (2012). For the effect of green tea supplementation, cholesterol, TG, LDL, HDL, VLDL, GOT and GPT values were observed to be lower when Green tea was added with $1.5 \%$. Thus, the addition of green tea can be considered as a managing technique to treat the stress of the density through nutrition. As for the interaction, we note that the highest increase in cholesterol, LDL, albumin, and GPT occurred was at $52 \mathrm{bird} / \mathrm{m}^{2}$ with $0 \%$ green tea. Stress as a result of crowding forces the bird to live in an insufficient area for movement which affects the behavior of the birds (Al-Hamed, 2010 ; Sahin et al., 2016). This leads to increased mortality due to diseases associated with low immunity and low natural body resistance (Al-Sheikhli, 2003 ; Tabler et al., 2004 ; Al-Naimi, 2018). Also, the emergence of hostility and increased incidence of cannibalism calculated among excluded individuals, but the addition of green tea, especially the concentration 1.0 and $1.5 \%$ had reduced this effect and enhanced the immunity of birds and raise the health level of birds which reflected in the performance of birds in general. 
Mesopotamia J. of Agric.

Vol. (48) No. (1) 2020
ISSN: $2224-9796$ (Online)

ISSN: 1815 - 316 X (Print)

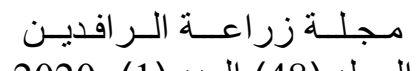

المجلد (48) العدد (1) 2020

Table (1): Effect of density and different levels of green tea and their interaction on body weight of quail.

\begin{tabular}{|c|c|c|c|c|c|c|}
\hline \multicolumn{2}{|c|}{$\overbrace{\text { Treatments }}^{\text {Weeks }}$} & $\begin{array}{c}\text { Initial body } \\
\text { weight ( } 2 \text { week) }\end{array}$ & $\begin{array}{l}\text { Final body } \\
\text { weight }\left(7^{\text {th }}\right)\end{array}$ & $\begin{array}{l}\text { Weight } \\
\text { gain }\end{array}$ & $\begin{array}{c}\text { Feed } \\
\text { consumption }\end{array}$ & $\begin{array}{c}\text { Feed } \\
\text { conversion }\end{array}$ \\
\hline \multicolumn{7}{|c|}{ Effect of Density $\left(\mathrm{bird} / \mathrm{m}^{2}\right)$ : } \\
\hline \multicolumn{2}{|c|}{44} & 24.16 & $\begin{array}{c}225.29 \mathrm{a} \\
\pm 1.99 \\
\end{array}$ & $\begin{array}{c}201.13 \mathrm{a} \\
\pm 2.29\end{array}$ & $\begin{array}{c}628.34 \mathrm{ab} \\
\pm 7.90 \\
\end{array}$ & $\begin{array}{l}3.12 \mathrm{~b} \\
\pm 0.05 \\
\end{array}$ \\
\hline \multicolumn{2}{|c|}{52} & 21.21 & $\begin{array}{c}216.84 \mathrm{~b} \\
\pm 3.75\end{array}$ & $\begin{array}{c}195.63 \mathrm{ab} \\
\pm 3.54\end{array}$ & $\begin{array}{c}618.27 \mathrm{~b} \\
\pm 9.59\end{array}$ & $\begin{array}{l}3.14 \mathrm{~b} \\
\pm 0.05\end{array}$ \\
\hline \multicolumn{2}{|c|}{60} & 22.04 & $\begin{array}{c}212.91 \mathrm{~b} \\
\pm 3.84\end{array}$ & $\begin{array}{c}190.87 \mathrm{~b} \\
\pm 2.47\end{array}$ & $\begin{array}{c}649.72 \mathrm{a} \\
\pm 6.77\end{array}$ & $\begin{array}{l}3.40 \mathrm{a} \\
\pm 0.06 \\
\end{array}$ \\
\hline \multicolumn{7}{|c|}{ Effect of Tea $(\%)$ : } \\
\hline \multicolumn{2}{|c|}{0.0} & 23.92 & $\begin{array}{c}218.49 \mathrm{~b} \\
\pm 1.79\end{array}$ & $\begin{array}{c}194.57 \mathrm{a} \\
\pm 2.47 \\
\end{array}$ & $\begin{array}{c}623.58 \mathrm{a} \\
\pm 9.19 \\
\end{array}$ & $\begin{array}{l}3.25 \mathrm{a} \\
\pm 0.09 \\
\end{array}$ \\
\hline \multicolumn{2}{|c|}{0.5} & 25.44 & $\begin{array}{c}224.29 \mathrm{a} \\
\pm 2.44\end{array}$ & $\begin{array}{c}198.85 \mathrm{a} \\
\pm 4.22\end{array}$ & $\begin{array}{l}637.20 \mathrm{a} \\
\pm 14.69\end{array}$ & $\begin{array}{l}3.20 \mathrm{a} \\
\pm 0.09\end{array}$ \\
\hline \multicolumn{2}{|c|}{1.0} & 25.44 & $\begin{array}{c}223.29 \mathrm{a} \\
\pm 3.78\end{array}$ & $\begin{array}{c}197.88 \mathrm{a} \\
\pm 2.42\end{array}$ & $\begin{array}{l}628.19 \mathrm{a} \\
\pm 8.05\end{array}$ & $\begin{array}{l}3.17 \mathrm{~b} \\
\pm 0.07\end{array}$ \\
\hline \multicolumn{2}{|c|}{1.5} & 25.82 & $\begin{array}{c}228.35 \mathrm{a} \\
\pm 3.51 \\
\end{array}$ & $\begin{array}{c}200.53 \mathrm{a} \\
\pm 4.94\end{array}$ & $\begin{array}{c}639.46 \mathrm{a} \\
\pm 25.69\end{array}$ & $\begin{array}{l}3.18 \mathrm{~b} \\
\pm 0.18\end{array}$ \\
\hline \multicolumn{7}{|c|}{ Effect of Interaction Between Density (bird $\left./ \mathrm{m}^{2}\right)$ and Tea $(\%)$ : } \\
\hline \multirow{4}{*}{$\begin{array}{c}44 \\
\left(\mathrm{bird} / \mathrm{m}^{2}\right)\end{array}$} & $0.0 \%$ & 23.46 & $\begin{array}{c}204.24 \mathrm{c} \\
\pm 2.44 \\
\end{array}$ & $\begin{array}{c}180.78 \mathrm{~d} \\
\pm 4.94 \\
\end{array}$ & $\begin{array}{c}614.79 \mathrm{~b} \\
\pm 25.69 \\
\end{array}$ & $\begin{array}{l}3.40 \mathrm{a} \\
\pm 0.18 \\
\end{array}$ \\
\hline & $0.5 \%$ & 23.27 & $\begin{array}{c}235.60 \mathrm{a} \\
\pm 3.78 \\
\end{array}$ & $\begin{array}{c}212.33 \mathrm{a} \\
. \pm 4.30\end{array}$ & $\begin{array}{c}624.07 \mathrm{~b} \\
\pm 6.63\end{array}$ & $\begin{array}{l}2.93 \mathrm{~d} \\
\pm 0.08\end{array}$ \\
\hline & $1.0 \%$ & 21.61 & $\begin{array}{c}225.89 \mathrm{~b} \\
\pm 3.59 \\
\end{array}$ & $\begin{array}{c}204.20 \mathrm{ab} \\
\pm 2.63 \\
\end{array}$ & $\begin{array}{c}633.14 \mathrm{~b} \\
\pm 12.84 \\
\end{array}$ & $\begin{array}{l}3.01 \mathrm{~d} \\
\pm 0.03 \\
\end{array}$ \\
\hline & $1.5 \%$ & 25.30 & $\begin{array}{c}232.54 \mathrm{a} \\
\pm 5.50\end{array}$ & $\begin{array}{c}207.24 \mathrm{~b} \\
\pm 2.59\end{array}$ & $\begin{array}{c}641.36 \mathrm{ab} \\
\pm 15.74\end{array}$ & $\begin{array}{l}3.09 \mathrm{~d} \\
\pm 0.03 \\
\end{array}$ \\
\hline \multirow{4}{*}{$\begin{array}{c}52 \\
\left(\mathrm{bird} / \mathrm{m}^{2}\right)\end{array}$} & $0.0 \%$ & 24.77 & $\begin{array}{l}209.11 \mathrm{c} \\
\pm 5.16\end{array}$ & $\begin{array}{c}184.34 \mathrm{~d} \\
\pm 3.84\end{array}$ & $\begin{array}{c}617.85 \mathrm{~b} \\
\pm 6.60\end{array}$ & $\begin{array}{l}3.35 \mathrm{a} \\
\pm 0.07\end{array}$ \\
\hline & $0.5 \%$ & 25.95 & $\begin{array}{l}216.6 \mathrm{bc} \\
\pm 10.54\end{array}$ & $\begin{array}{c}190.63 \mathrm{c} \\
\pm 3.43\end{array}$ & $\begin{array}{c}603.16 \mathrm{~b} \\
\pm 33.45\end{array}$ & $\begin{array}{l}3.16 \mathrm{c} \\
\pm 0.04\end{array}$ \\
\hline & $1.0 \%$ & 23.24 & $\begin{array}{c}216.2 \mathrm{bc} \\
\pm 5.25\end{array}$ & $\begin{array}{l}193.0 \mathrm{c} \\
\pm 3.91 \\
\end{array}$ & $\begin{array}{c}603.99 \mathrm{~b} \\
\pm 10.04 \\
\end{array}$ & $\begin{array}{l}3.13 \mathrm{c} \\
\pm 0.08 \\
\end{array}$ \\
\hline & $1.5 \%$ & 22.22 & $\begin{array}{c}232.45 \mathrm{a} \\
\pm 4.05\end{array}$ & $\begin{array}{c}210.23 \mathrm{a} \\
\pm 8.21\end{array}$ & $\begin{array}{c}648.06 \mathrm{ab} \\
\pm 11.61\end{array}$ & $\begin{array}{l}3.08 \mathrm{~d} \\
\pm 0.04\end{array}$ \\
\hline \multirow{4}{*}{$\begin{array}{c}60 \\
\left(\mathrm{bird} / \mathrm{m}^{2}\right)\end{array}$} & $0.0 \%$ & 22.51 & $\begin{array}{c}207.12 \mathrm{c} \\
\pm 2.31\end{array}$ & $\begin{array}{c}184.61 \mathrm{~d} \\
\pm 4.29\end{array}$ & $\begin{array}{c}638.09 \mathrm{ab} \\
\pm 10.89\end{array}$ & $\begin{array}{l}3.46 \mathrm{a} \\
\pm 0.09\end{array}$ \\
\hline & $0.5 \%$ & 23.10 & $\begin{array}{c}210.7 \mathrm{bc} \\
\pm 5.01\end{array}$ & $\begin{array}{c}187.60 \mathrm{~d} \\
\pm 8.54 \\
\end{array}$ & $\begin{array}{c}684.37 \mathrm{a} \\
\pm 5.81 \\
\end{array}$ & $\begin{array}{l}3.45 \mathrm{a} \\
\pm 0.07 \\
\end{array}$ \\
\hline & $1.0 \%$ & 21.65 & $\begin{array}{c}213.8 \mathrm{bc} \\
\pm 1.46\end{array}$ & $\begin{array}{c}192.15 \mathrm{c} \\
\pm 2.49\end{array}$ & $\begin{array}{c}627.45 \mathrm{~b} \\
\pm 10.58\end{array}$ & $\begin{array}{l}3.26 \mathrm{~b} \\
\pm 0.06\end{array}$ \\
\hline & $1.5 \%$ & 23.58 & $\begin{array}{c}215.7 \mathrm{bc} \\
\pm 3.81\end{array}$ & $\begin{array}{c}192.12 \mathrm{c} \\
\pm 1.77\end{array}$ & $\begin{array}{c}628.98 \mathrm{~b} \\
\pm 7.90\end{array}$ & $\begin{array}{l}3.27 \mathrm{~b} \\
\pm 0.02\end{array}$ \\
\hline
\end{tabular}

- The above values represent mean \pm standard error.

- Values that have different letters vertically indicate a significant difference at $(\mathrm{p} \leq 0.05)$. 
Mesopotamia J. of Agric.

Vol. (48) No. (1) 2020
ISSN: $2224-9796$ (Online)

ISSN: 1815 - 316 X (Print)

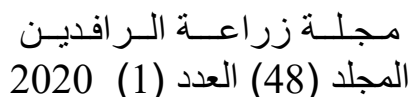

Table (2): Effect of density and different levels of green tea and their interaction on mortality $\%$, index production, and coefficient production at $7^{\text {th }}$ week aged quail.

\begin{tabular}{|c|c|c|c|c|}
\hline Treatment & Weeks & Mortality\% & production index & production coefficient \\
\hline \multicolumn{5}{|c|}{ Effect of Density (bird/m²): } \\
\hline \multicolumn{2}{|c|}{44} & $4.17 \pm 1.20 \mathrm{~b}$ & $14.12 \pm 0.50 \mathrm{a}$ & $6.18 \pm 1.39 \mathrm{a}$ \\
\hline \multicolumn{2}{|c|}{52} & $7.50 \pm 1.34 \mathrm{~b}$ & $13.04 \pm 0.42 \mathrm{a}$ & $5.76 \pm 0.62 \mathrm{~b}$ \\
\hline \multicolumn{2}{|c|}{60} & $12.08 \pm 1.25 \mathrm{a}$ & $10.09 \pm 0.34 \mathrm{~b}$ & $4.94 \pm 0.55 \mathrm{~b}$ \\
\hline \multicolumn{5}{|c|}{ Effect of green Tea $(\%)$ : } \\
\hline \multicolumn{2}{|c|}{0.0} & $10.56 \pm 1.19 \mathrm{a}$ & $12.27 \pm 0.80 \mathrm{a}$ & $5.35 \pm 0.85 \mathrm{a}$ \\
\hline \multicolumn{2}{|c|}{0.5} & $8.33 \pm 1.19 \mathrm{ab}$ & $13.11 \pm 1.39 \mathrm{a}$ & $5.67 \pm 1.28 \mathrm{a}$ \\
\hline \multicolumn{2}{|c|}{1.0} & $7.22 \pm 1.93 \mathrm{ab}$ & $13.34 \pm 0.22 \mathrm{a}$ & $5.79 \pm 1.01 \mathrm{a}$ \\
\hline \multicolumn{2}{|c|}{$\begin{array}{r}1.5 \\
\end{array}$} & $5.56 \pm 1.09 \mathrm{~b}$ & $13.84 \pm 0.87 \mathrm{a}$ & $5.96 \pm 0.75 \mathrm{a}$ \\
\hline \multicolumn{5}{|c|}{ Effect of Interaction Between Density (bird $\left./ \mathrm{m}^{2}\right)$ and Green Tea (\%): } \\
\hline \multirow{4}{*}{$\begin{array}{c}44 \\
\left(\mathrm{bird} / \mathrm{m}^{2}\right)\end{array}$} & $0.0 \%$ & $5.00 \pm 1.20 \mathrm{c}$ & $11.65 \pm 1.22 \mathrm{~b}$ & $5.51 \pm 0.91 \mathrm{~b}$ \\
\hline & $0.5 \%$ & $3.34 \pm 1.03 \mathrm{~d}$ & $15.86 \pm 0.22 \mathrm{a}$ & $7.00 \pm 0.61 \mathrm{a}$ \\
\hline & $1.0 \%$ & $3.34 \pm 1.83 \mathrm{~d}$ & $14.80 \pm 0.44 \mathrm{a}$ & $4.73 \pm 0.51 \mathrm{c}$ \\
\hline & $1.5 \%$ & $3.34 \pm 1.91 \mathrm{~d}$ & $14.85 \pm 0.90 \mathrm{a}$ & $6.48 \pm 1.32 \mathrm{ab}$ \\
\hline \multirow{4}{*}{$\begin{array}{c}52 \\
\left(\mathrm{bird} / \mathrm{m}^{2}\right)\end{array}$} & $0.0 \%$ & $10.00 \pm 3.19 \mathrm{~b}$ & $11.47 \pm 1.70 \mathrm{~b}$ & $4.95 \pm 0.58 \mathrm{c}$ \\
\hline & $0.5 \%$ & $8.33 \pm 2.72 \mathrm{bc}$ & $12.82 \pm 0.56 \mathrm{ab}$ & $5.53 \pm 0.37 \mathrm{~b}$ \\
\hline & $1.0 \%$ & $6.67 \pm 3.09 \mathrm{c}$ & $13.16 \pm 0.99 \mathrm{a}$ & $5.75 \pm 0.85 \mathrm{~b}$ \\
\hline & $1.5 \%$ & $5.00 \pm 1.81 \mathrm{c}$ & $14.63 \pm 0.43 \mathrm{a}$ & $6.48 \pm 0.90 \mathrm{ab}$ \\
\hline \multirow{4}{*}{$\begin{array}{c}60 \\
\left(\mathrm{bird} / \mathrm{m}^{2}\right)\end{array}$} & $0.0 \%$ & $16.67 \pm 1.67 \mathrm{a}$ & $10.18 \pm 0.34 \mathrm{~b}$ & $4.45 \pm 0.24 \mathrm{c}$ \\
\hline & $0.5 \%$ & $11.67 \pm 1.55 \mathrm{~b}$ & $11.01 \pm 0.39 \mathrm{~b}$ & $4.80 \pm 0.86 \mathrm{c}$ \\
\hline & $1.0 \%$ & $11.67 \pm 3.19 \mathrm{~b}$ & $11.82 \pm 0.31 \mathrm{~b}$ & $5.21 \pm 1.17 \mathrm{~b}$ \\
\hline & $1.5 \%$ & $8.33 \pm 1.60 \mathrm{bc}$ & $12.34 \pm 0.40 \mathrm{~b}$ & $5.39 \pm 1.24 \mathrm{~b}$ \\
\hline
\end{tabular}

- The above values represent mean \pm standard error .

- Values that have different letters vertically indicate a significant difference at $(\mathrm{p} \leq 0.05)$. 
Mesopotamia J. of Agric.

Vol. (48) No. (1) 2020
ISSN: 2224 - 9796 (Online)

ISSN: 1815 - 316 X (Print)
مـجلــة زر اعــة الــر افديـن

المجلد (48) العدد (1) 2020

Table (3):Effect of density and different levels of green tea and their interaction on lipid profile parameters of quail at 49 days age.

\begin{tabular}{|c|c|c|c|c|c|c|}
\hline \multicolumn{2}{|c|}{ Treatments } & $\begin{array}{c}\text { Cholesterol } \\
\mathrm{mg} / \mathrm{dl}\end{array}$ & $\begin{array}{c}\text { triglycerides } \\
\mathrm{mg} / \mathrm{dl}\end{array}$ & $\begin{array}{l}\text { LDL } \\
\mathrm{mg} / \mathrm{dl}\end{array}$ & $\begin{array}{l}\mathrm{HDL} \\
\mathrm{mg} / \mathrm{dl}\end{array}$ & $\begin{array}{l}\text { VLDL } \\
\mathrm{mg} / \mathrm{dl}\end{array}$ \\
\hline \multicolumn{7}{|c|}{ Effect of Density $\left(\mathrm{bird} / \mathrm{m}^{2}\right)$ : } \\
\hline \multicolumn{2}{|c|}{44} & $\begin{array}{l}5.27 \mathrm{a} \\
\pm 0.21 \\
\end{array}$ & $\begin{array}{l}5.90 \mathrm{a} \\
\pm 0.26 \\
\end{array}$ & $\begin{array}{c}3.13 \mathrm{~b} \\
\pm 0.8 \\
\end{array}$ & $\begin{array}{l}1.23 \mathrm{a} \\
\pm 0.05 \\
\end{array}$ & $\begin{array}{l}1.19 \mathrm{a} \\
\pm 0.04 \\
\end{array}$ \\
\hline \multicolumn{2}{|l|}{52} & $\begin{array}{l}4.96 \mathrm{a} \\
\pm 0.37\end{array}$ & $\begin{array}{l}6.28 \mathrm{a} \\
\pm 0.44\end{array}$ & $\begin{array}{l}3.97 \mathrm{a} \\
\pm 0.21\end{array}$ & $\begin{array}{l}1.17 \mathrm{a} \\
\pm 0.04\end{array}$ & $\begin{array}{l}1.25 \mathrm{a} \\
\pm 0.06\end{array}$ \\
\hline \multicolumn{2}{|c|}{60} & $\begin{array}{l}5.78 \mathrm{a} \\
\pm 0.34 \\
\end{array}$ & $\begin{array}{l}6.15 \mathrm{a} \\
\pm 0.50 \\
\end{array}$ & $\begin{array}{c}3.72 \mathrm{ab} \\
\pm 0.32 \\
\end{array}$ & $\begin{array}{l}1.32 \mathrm{a} \\
\pm 0.10 \\
\end{array}$ & $\begin{array}{l}1.20 \mathrm{a} \\
\pm 0.08 \\
\end{array}$ \\
\hline \multicolumn{7}{|c|}{ Effect of Tea $(\%)$ : } \\
\hline \multicolumn{2}{|c|}{0.0} & $\begin{array}{l}5.91 \mathrm{a} \\
\pm 0.35 \\
\end{array}$ & $\begin{array}{l}7.04 \mathrm{a} \\
\pm 0.46 \\
\end{array}$ & $\begin{array}{l}4.12 \mathrm{a} \\
\pm 0.37 \\
\end{array}$ & $\begin{array}{l}1.00 \mathrm{c} \\
\pm 0.12 \\
\end{array}$ & $\begin{array}{l}1.37 \mathrm{a} \\
\pm 0.05 \\
\end{array}$ \\
\hline \multicolumn{2}{|c|}{0.5} & $\begin{array}{l}5.97 \mathrm{a} \\
\pm 8.30\end{array}$ & $\begin{array}{l}6.07 \mathrm{a} \\
\pm 0.55 \\
\end{array}$ & $\begin{array}{l}3.70 \mathrm{a} \\
\pm 0.55 \\
\end{array}$ & $\begin{array}{l}1.33 \mathrm{~b} \\
\pm 0.09\end{array}$ & $\begin{array}{l}1.22 \mathrm{a} \\
\pm 0.07\end{array}$ \\
\hline \multicolumn{2}{|c|}{1.0} & $\begin{array}{c}4.88 \mathrm{ab} \\
\pm 0.64\end{array}$ & $\begin{array}{l}6.60 \mathrm{a} \\
\pm 0.88\end{array}$ & $\begin{array}{l}3.91 \mathrm{a} \\
\pm 0.06\end{array}$ & $\begin{array}{l}1.45 \mathrm{a} \\
\pm 0.06\end{array}$ & $\begin{array}{c}1.32 \mathrm{a} \\
\pm 0.3\end{array}$ \\
\hline \multicolumn{2}{|c|}{1.5} & $\begin{array}{l}4.59 \mathrm{~b} \\
\pm 0.61 \\
\end{array}$ & $\begin{array}{l}4.73 \mathrm{~b} \\
\pm 1.24 \\
\end{array}$ & $\begin{array}{l}2.69 \mathrm{~b} \\
\pm 0.32 \\
\end{array}$ & $\begin{array}{c}1.17 \mathrm{bc} \\
\pm 0.05\end{array}$ & $\begin{array}{l}0.95 \mathrm{~b} \\
\pm 0.10 \\
\end{array}$ \\
\hline \multicolumn{7}{|c|}{ Effect of Interaction Between Density $\left(\mathrm{bird} / \mathrm{m}^{2}\right)$ and Tea $(\%)$ : } \\
\hline \multirow{4}{*}{$\begin{array}{c}44 \\
\left(\operatorname{bird} / \mathrm{m}^{2}\right)\end{array}$} & $0.0 \%$ & $\begin{array}{l}6.60 \mathrm{ab} \\
\pm 0.79\end{array}$ & $\begin{array}{l}5.70 \mathrm{a} \\
\pm 0.63\end{array}$ & $\begin{array}{c}3.50 \mathrm{ab} \\
\pm 0.61\end{array}$ & $\begin{array}{l}1.20 \mathrm{bc} \\
\pm 0.03\end{array}$ & $\begin{array}{l}1.16 \mathrm{~b} \\
\pm 0.05\end{array}$ \\
\hline & $0.5 \%$ & $\begin{array}{l}5.50 \mathrm{ab} \\
\pm 0.61 \\
\end{array}$ & $\begin{array}{l}7.10 \mathrm{a} \\
\pm 1.24 \\
\end{array}$ & $\begin{array}{c}2.98 \mathrm{bc} \\
\pm 0.32 \\
\end{array}$ & $\begin{array}{l}1.10 \mathrm{bc} \\
\pm 0.05\end{array}$ & $\begin{array}{l}1.42 \mathrm{a} \\
\pm 0.10 \\
\end{array}$ \\
\hline & $1.0 \%$ & $\begin{array}{c}.67 \mathrm{ab} \\
\pm 0.30\end{array}$ & $\begin{array}{l}5.90 \mathrm{a} \\
\pm 0.55\end{array}$ & $\begin{array}{l}3.32 \mathrm{~b} \\
\pm 0.55\end{array}$ & $\begin{array}{l}1.40 \mathrm{~b} \\
\pm 0.09\end{array}$ & $\begin{array}{l}1.18 \mathrm{~b} \\
\pm 0.07\end{array}$ \\
\hline & $1.5 \%$ & $\begin{array}{l}4.30 \mathrm{~b} \\
\pm 0.64\end{array}$ & $\begin{array}{l}4.90 \mathrm{a} \\
\pm 0.88 \\
\end{array}$ & $\begin{array}{c}2.72 \mathrm{bc} \\
\pm 0.06\end{array}$ & $\begin{array}{l}1.20 \mathrm{bc} \\
\pm 0.06\end{array}$ & $\begin{array}{l}0.98 \mathrm{c} \\
\pm 0.13 \\
\end{array}$ \\
\hline \multirow{4}{*}{$\begin{array}{c}52 \\
\left(\mathrm{bird} / \mathrm{m}^{2}\right)\end{array}$} & $0.0 \%$ & $\begin{array}{c}5.50 \mathrm{ab} \\
\pm 0.62\end{array}$ & $\begin{array}{l}6.40 \mathrm{a} \\
\pm 1.17 \\
\end{array}$ & $\begin{array}{c}4.02 \mathrm{ab} \\
\pm 0.82\end{array}$ & $\begin{array}{l}1.10 \mathrm{bc} \\
\pm 0.05\end{array}$ & $\begin{array}{c}1.27 \mathrm{ab} \\
\pm 0.19\end{array}$ \\
\hline & $0.5 \%$ & $\begin{array}{l}5.42 \mathrm{ab} \\
\pm 1.07\end{array}$ & $\begin{array}{l}6.80 \mathrm{a} \\
\pm 1.10 \\
\end{array}$ & $\begin{array}{c}4.54 \mathrm{ab} \\
\pm 0.33 \\
\end{array}$ & $\begin{array}{l}0.90 \mathrm{c} \\
\pm 0.07 \\
\end{array}$ & $\begin{array}{l}1.36 \mathrm{a} \\
\pm 0.09 \\
\end{array}$ \\
\hline & $1.0 \%$ & $\begin{array}{l}4.50 \mathrm{~b} \\
\pm 0.68 \\
\end{array}$ & $\begin{array}{l}7.30 \mathrm{a} \\
\pm 0.82 \\
\end{array}$ & $\begin{array}{c}4.54 \mathrm{ab} \\
\pm 0.60 \\
\end{array}$ & $\begin{array}{c}1.56 \mathrm{ab} \\
\pm 0.27 \\
\end{array}$ & $\begin{array}{l}1.46 \mathrm{a} \\
\pm 0.10 \\
\end{array}$ \\
\hline & $0.5 \%$ & $\begin{array}{l}4.40 \mathrm{~b} \\
\pm 0.29\end{array}$ & $\begin{array}{l}4.60 \mathrm{a} \\
\pm 0.44\end{array}$ & $\begin{array}{c}2.78 \mathrm{bc} \\
\pm 0.11\end{array}$ & $\begin{array}{l}1.10 \mathrm{bc} \\
\pm 0.08\end{array}$ & $\begin{array}{l}0.92 \mathrm{c} \\
\pm 0.04\end{array}$ \\
\hline \multirow{4}{*}{$\begin{array}{c}60 \\
\left(\mathrm{bird} / \mathrm{m}^{2}\right)\end{array}$} & $0.0 \%$ & $\begin{array}{l}6.80 \mathrm{a} \\
\pm 0.88\end{array}$ & $\begin{array}{l}7.21 \mathrm{a} \\
\pm 0.53\end{array}$ & $\begin{array}{l}4.84 \mathrm{a} \\
\pm 0.83\end{array}$ & $\begin{array}{l}1.00 \mathrm{c} \\
\pm 0.14\end{array}$ & $\begin{array}{l}1.33 \mathrm{a} \\
\pm 0.08\end{array}$ \\
\hline & $0.5 \%$ & $\begin{array}{l}5.07 \mathrm{ab} \\
\pm 0.87 \\
\end{array}$ & $\begin{array}{l}4.70 \mathrm{a} \\
\pm 1.22 \\
\end{array}$ & $\begin{array}{l}2.56 \mathrm{c} \\
\pm 0.34 \\
\end{array}$ & $\begin{array}{l}1.2 \mathrm{bc} \\
\pm 0.32 \\
\end{array}$ & $\begin{array}{l}0.95 \mathrm{c} \\
\pm 0.02 \\
\end{array}$ \\
\hline & $1.5 \%$ & $\begin{array}{l}5.80 \mathrm{ab} \\
\pm 0.52 \\
\end{array}$ & $\begin{array}{l}6.10 \mathrm{a} \\
\pm 0.52 \\
\end{array}$ & $\begin{array}{c}3.58 \mathrm{ab} \\
\pm 0.48 \\
\end{array}$ & $\begin{array}{l}1.69 \mathrm{a} \\
\pm 0.40 \\
\end{array}$ & $\begin{array}{c}1.22 \mathrm{ab} \\
\pm 0.17 \\
\end{array}$ \\
\hline & $1.0 \%$ & $\begin{array}{c}5.47 \mathrm{ab} \\
\pm 0.34\end{array}$ & $\begin{array}{l}6.60 \mathrm{a} \\
\pm 0.93\end{array}$ & $\begin{array}{c}3.88 \mathrm{ab} \\
\pm 0.78\end{array}$ & $\begin{array}{l}1.40 \mathrm{~b} \\
\pm 0.04\end{array}$ & $\begin{array}{l}1.32 \mathrm{a} \\
\pm 0.02\end{array}$ \\
\hline
\end{tabular}

- The above values represent mean \pm standard error.

- Values that have different letters vertically indicate a significant difference at $(p \leq 0.05)$. 
Mesopotamia J. of Agric.

Vol. (48) No. (1) 2020
ISSN: $2224-9796$ (Online)

ISSN: 1815 - 316 X (Print)

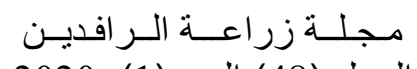

المجلد (48) العدد (1) 2020

Table (4): Effect of density and different levels of green tea and their interaction on some blood biochemical parameters of quail at 49 days age.

\begin{tabular}{|c|c|c|c|c|c|c|}
\hline \multicolumn{2}{|c|}{ Treatments } & $\begin{array}{l}\text { Glucose } \\
(\mathrm{mg} / \mathrm{dl})\end{array}$ & $\begin{array}{l}\text { Protein } \\
\mathrm{g} / 1\end{array}$ & $\begin{array}{l}\text { Albumen } \\
\mathrm{g} / \mathrm{l}\end{array}$ & $\begin{array}{l}\text { GPT } \\
\text { IU/L }\end{array}$ & $\begin{array}{l}\text { GOT } \\
\text { IU/L }\end{array}$ \\
\hline \multicolumn{7}{|c|}{ Effect of Density $\left(\mathrm{bird} / \mathrm{m}^{2}\right)$ : } \\
\hline \multicolumn{2}{|c|}{44} & $\begin{array}{c}223.67 \mathrm{c} \\
\pm 3.74 \\
\end{array}$ & $\begin{array}{l}3.89 \mathrm{~b} \\
\pm 0.21 \\
\end{array}$ & $\begin{array}{l}2.33 \mathrm{a} \\
\pm 0.07 \\
\end{array}$ & $\begin{array}{c}17.38 \mathrm{a} \\
\pm 0.66 \\
\end{array}$ & $\begin{array}{c}13.50 \mathrm{a} \\
\pm 0.62 \\
\end{array}$ \\
\hline \multicolumn{2}{|c|}{52} & $\begin{array}{c}229.33 \mathrm{~b} \\
\pm 9.07\end{array}$ & $\begin{array}{c}4.45 \mathrm{ab} \\
\pm 0.38 \\
\end{array}$ & $\begin{array}{l}2.50 \mathrm{a} \\
\pm 0.11 \\
\end{array}$ & $\begin{array}{c}16.59 \mathrm{a} \\
\pm 1.17 \\
\end{array}$ & $\begin{array}{c}14.00 \mathrm{a} \\
\pm 1.35 \\
\end{array}$ \\
\hline \multicolumn{2}{|c|}{60} & $\begin{array}{c}235.82 \mathrm{a} \\
\pm 5.00\end{array}$ & $\begin{array}{l}5.00 \mathrm{a} \\
\pm 0.27 \\
\end{array}$ & $\begin{array}{l}2.44 \mathrm{a} \\
\pm 0.14 \\
\end{array}$ & $\begin{array}{c}17.00 \mathrm{a} \\
\pm 1.34 \\
\end{array}$ & $\begin{array}{c}14.80 \mathrm{a} \\
\pm 1.16 \\
\end{array}$ \\
\hline \multicolumn{7}{|c|}{ Effect of Tea $(\%)$ : } \\
\hline \multicolumn{2}{|c|}{0.0} & $\begin{array}{c}235.65 \mathrm{~b} \\
\pm 4.43\end{array}$ & $\begin{array}{l}4.10 \mathrm{a} \\
\pm 0.38\end{array}$ & $\begin{array}{l}2.15 \mathrm{~b} \\
\pm 0.12\end{array}$ & $\begin{array}{c}19.72 \mathrm{a} \\
\pm 1.00\end{array}$ & $\begin{array}{c}17.33 \mathrm{a} \\
\pm 6.64\end{array}$ \\
\hline \multicolumn{2}{|c|}{0.5} & $\begin{array}{c}169.89 \mathrm{c} \\
\pm 3.33\end{array}$ & $\begin{array}{l}4.10 \mathrm{a} \\
\pm 0.48\end{array}$ & $\begin{array}{l}2.30 \mathrm{~b} \\
\pm 0.08\end{array}$ & $\begin{array}{c}18.00 \mathrm{ab} \\
\pm 2.15\end{array}$ & $\begin{array}{c}15.33 \mathrm{a} \\
\pm 1.99\end{array}$ \\
\hline \multicolumn{2}{|c|}{1.0} & $\begin{array}{c}246.89 \mathrm{a} \\
\pm 2.28\end{array}$ & $\begin{array}{l}5.08 \mathrm{a} \\
\pm 0.40\end{array}$ & $\begin{array}{l}2.50 \mathrm{ab} \\
\pm 0.11\end{array}$ & $\begin{array}{c}16.45 \mathrm{bc} \\
\pm 1.39\end{array}$ & $\begin{array}{c}13.01 \mathrm{~b} \\
\pm 2.14\end{array}$ \\
\hline \multicolumn{2}{|c|}{1.5} & $\begin{array}{c}239.00 \mathrm{~b} \\
\pm 2.28\end{array}$ & $\begin{array}{l}4.55 \mathrm{a} \\
\pm 0.16\end{array}$ & $\begin{array}{l}2.73 \mathrm{a} \\
\pm 0.50\end{array}$ & $\begin{array}{c}13.77 \mathrm{c} \\
\pm 2.41\end{array}$ & $\begin{array}{c}10.72 \mathrm{c} \\
\pm 1.37\end{array}$ \\
\hline \multicolumn{7}{|c|}{ Effect of Interaction Between Density (bird/ $/ \mathrm{m}^{2}$ )and Tea (\%): } \\
\hline \multirow{4}{*}{$\begin{array}{c}44 \\
\left(\mathrm{bird} / \mathrm{m}^{2}\right)\end{array}$} & $0.0 \%$ & $\begin{array}{c}224.00 \mathrm{~d} \\
\pm 3.23\end{array}$ & $\begin{array}{c}4.01 \mathrm{ab} \\
\pm 0.48\end{array}$ & $\begin{array}{c}2.20 \mathrm{bc} \\
\pm 0.08\end{array}$ & $\begin{array}{c}18.17 \mathrm{ab} \\
\pm 0.08\end{array}$ & $\begin{array}{c}16.0 \mathrm{ab} \\
\pm 1.99 \\
\end{array}$ \\
\hline & $0.5 \%$ & $\begin{array}{c}175.00 \mathrm{~g} \\
\pm 2.28\end{array}$ & $\begin{array}{c}4.00 \mathrm{ab} \\
\pm 0.40\end{array}$ & $\begin{array}{l}2.30 \mathrm{~b} \\
\pm 0.11\end{array}$ & $\begin{array}{c}21.00 \mathrm{a} \\
\pm 0.11\end{array}$ & $\begin{array}{l}18.00 \mathrm{a} \\
\pm 2.14\end{array}$ \\
\hline & $1.0 \%$ & $\begin{array}{c}250.67 \mathrm{a} \\
\pm 2.28\end{array}$ & $\begin{array}{c}4.35 \mathrm{ab} \\
\pm 1.16 \\
\end{array}$ & $\begin{array}{c}2.20 \mathrm{bc} \\
\pm 0.06\end{array}$ & $\begin{array}{c}16.00 \mathrm{~b} \\
\pm 0.06\end{array}$ & $\begin{array}{c}18.00 \mathrm{a} \\
\pm 1.35 \\
\end{array}$ \\
\hline & $1.5 \%$ & $\begin{array}{c}245.00 \mathrm{ab} \\
\pm 3.56\end{array}$ & $\begin{array}{l}3.20 \mathrm{~b} \\
\pm 1.05\end{array}$ & $\begin{array}{l}2.60 \mathrm{a} \\
\pm 0.45\end{array}$ & $\begin{array}{c}14.00 \mathrm{c} \\
\pm 0.45\end{array}$ & $\begin{array}{l}8.00 \mathrm{~d} \\
\pm 0.47\end{array}$ \\
\hline \multirow{4}{*}{$\begin{array}{c}52 \\
\left(\mathrm{bird} / \mathrm{m}^{2}\right)\end{array}$} & $0.0 \%$ & $\begin{array}{c}233.00 \mathrm{c} \\
\pm 1.51\end{array}$ & $\begin{array}{c}4.20 \mathrm{ab} \\
\pm 0.21\end{array}$ & $\begin{array}{l}2.00 \mathrm{c} \\
\pm 0.36\end{array}$ & $\begin{array}{c}21.00 \mathrm{a} \\
\pm 0.36\end{array}$ & $\begin{array}{c}19.00 \mathrm{a} \\
\pm 0.92\end{array}$ \\
\hline & $0.5 \%$ & $\begin{array}{c}202.00 \mathrm{f} \\
\pm 3.18 \\
\end{array}$ & $\begin{array}{c}4.10 \mathrm{ab} \\
\pm 0.35\end{array}$ & $\begin{array}{l}2.50 \mathrm{a} \\
\pm 0.11\end{array}$ & $\begin{array}{c}19.00 \mathrm{a} \\
\pm 0.11\end{array}$ & $\begin{array}{l}15.0 \mathrm{~b} \\
\pm 0.81\end{array}$ \\
\hline & $1.0 \%$ & $\begin{array}{c}243.33 \mathrm{ab} \\
\pm 1.35\end{array}$ & $\begin{array}{c}5.30 \mathrm{ab} \\
\pm 0.92 \\
\end{array}$ & $\begin{array}{l}2.80 \mathrm{a} \\
\pm 0.16 \\
\end{array}$ & $\begin{array}{c}16.35 \mathrm{~b} \\
\pm 0.16 \\
\end{array}$ & $\begin{array}{c}13.00 \mathrm{c} \\
\pm 1.04\end{array}$ \\
\hline & $1.5 \%$ & $\begin{array}{c}239.00 \mathrm{~b} \\
\pm 3.17\end{array}$ & $\begin{array}{c}4.21 \mathrm{ab} \\
\pm 0.30\end{array}$ & $\begin{array}{l}2.70 \mathrm{a} \\
\pm 0.33\end{array}$ & $\begin{array}{l}9.99 \mathrm{~d} \\
\pm 0.33\end{array}$ & $\begin{array}{l}9.00 \mathrm{~d} \\
\pm 0.94\end{array}$ \\
\hline \multirow{4}{*}{$\begin{array}{c}60 \\
\left(\mathrm{bird} / \mathrm{m}^{2}\right)\end{array}$} & $0.0 \%$ & $\begin{array}{c}249.96 \mathrm{a} \\
\pm 2.99\end{array}$ & $\begin{array}{c}4.10 \mathrm{ab} \\
\pm 0.28\end{array}$ & $\begin{array}{l}2.26 \mathrm{~b} \\
\pm 0.14\end{array}$ & $\begin{array}{c}20.00 \mathrm{a} \\
\pm 0.14\end{array}$ & $\begin{array}{l}17.0 \mathrm{a} \\
\pm 1.00\end{array}$ \\
\hline & $0.5 \%$ & $\begin{array}{c}213.67 \mathrm{e} \\
\pm 1.26 \\
\end{array}$ & $\begin{array}{c}4.20 \mathrm{ab} \\
\pm 0.38\end{array}$ & $\begin{array}{l}2.10 \mathrm{c} \\
\pm 0.02 \\
\end{array}$ & $\begin{array}{c}14.01 \mathrm{c} \\
\pm 0.02 \\
\end{array}$ & $\begin{array}{c}13.00 \mathrm{c} \\
\pm 1.21 \\
\end{array}$ \\
\hline & $1.0 \%$ & $\begin{array}{c}246.07 \mathrm{ab} \\
\pm 2.68\end{array}$ & $\begin{array}{l}5.60 \mathrm{a} \\
\pm 0.61\end{array}$ & $\begin{array}{l}2.50 \mathrm{a} \\
\pm 0.36\end{array}$ & $\begin{array}{c}17.00 \mathrm{ab} \\
\pm 0.36\end{array}$ & $\begin{array}{c}14.04 \mathrm{~b} \\
\pm 1.11\end{array}$ \\
\hline & $1.5 \%$ & $\begin{array}{c}233.00 \mathrm{c} \\
\pm 2.78\end{array}$ & $\begin{array}{l}6.23 \mathrm{a} \\
\pm 0.94\end{array}$ & $\begin{array}{l}2.90 \mathrm{a} \\
\pm 0.05\end{array}$ & $\begin{array}{c}17.00 \mathrm{ab} \\
\pm 0.05\end{array}$ & $\begin{array}{c}15.15 \mathrm{~b} \\
\pm 1.00\end{array}$ \\
\hline
\end{tabular}

- The above values represent mean \pm standard error.

- Values that have different letters vertically indicate a significant difference at $(p \leq 0.05)$. 


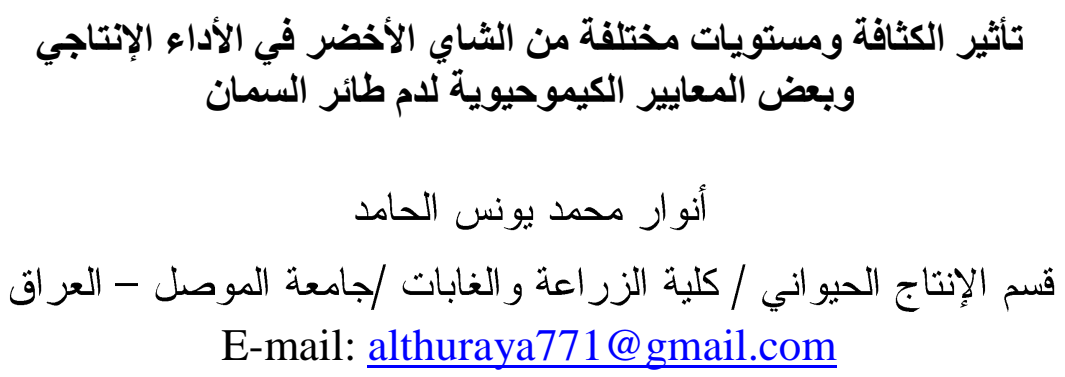

\section{الخلاصة}

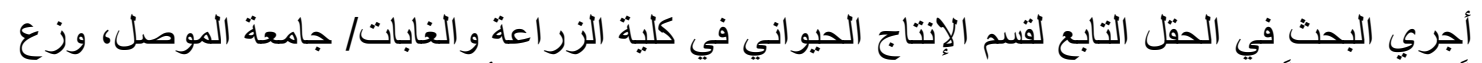

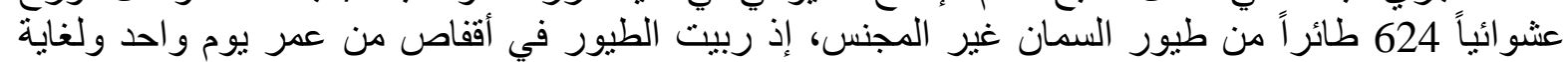

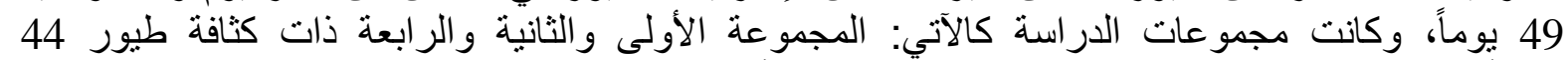

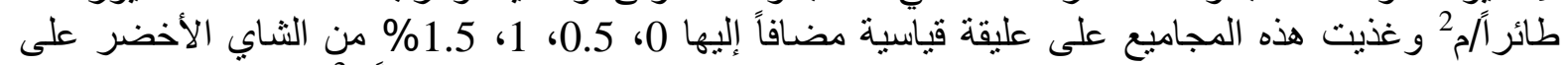

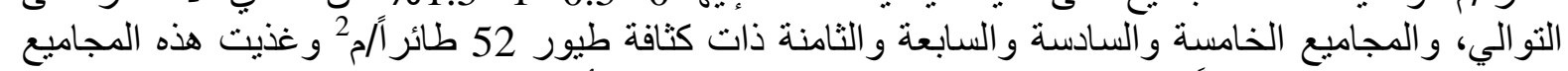

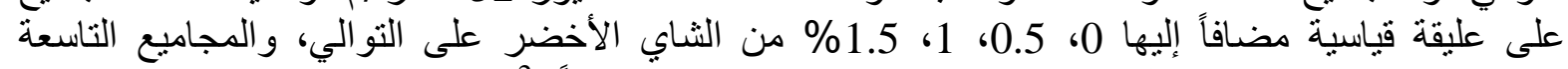

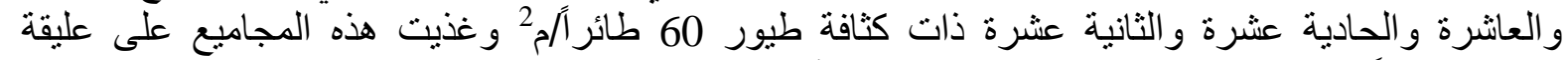

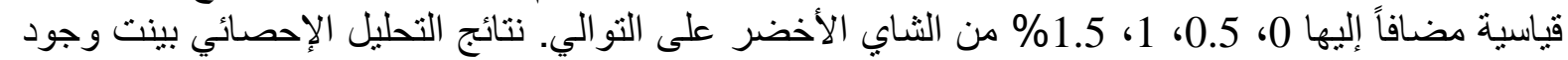

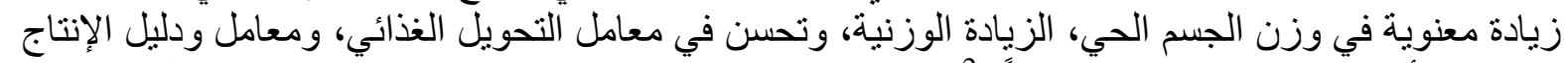

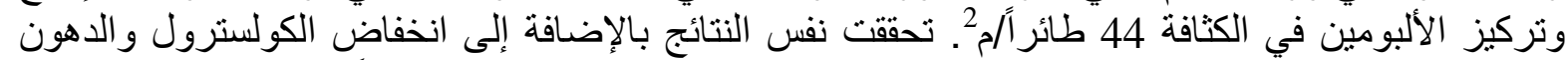

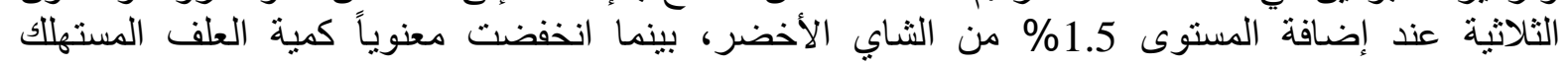

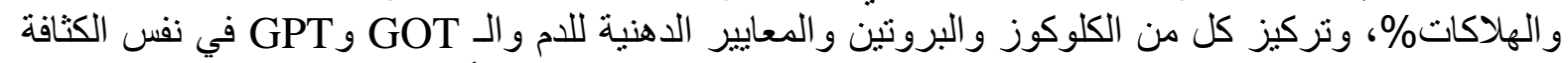

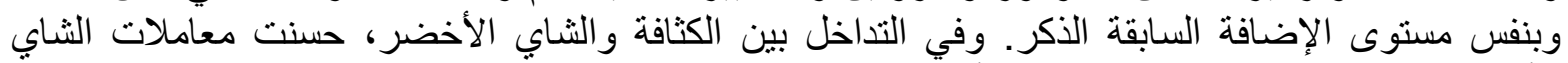

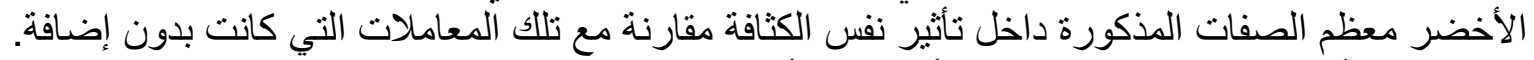

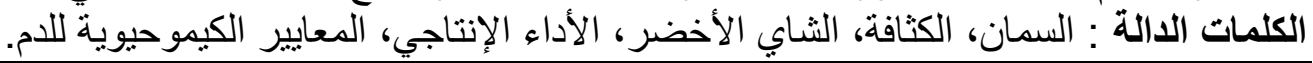

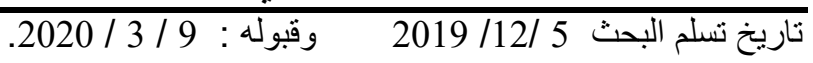

\section{REFERENCES}

Abdel-Azeem, F.A. (2010). The influence of different stoking density and sex on productive performance and some physiological treats of Japanese quail. Egypt. Poultry Science:30 (1):203-227.

Abou El-Ela, Salah El-Din (2005). Quail - Breeding- Caring - Nutrition - Projects. First edition, Faculty of Agriculture -Zagazig University.

Agrawal, S.K. ;S.K. Bbhanja ; S. Majumder and R. Narayan (2003) Effect of Floor Space on the Performance of Broiler Quails at Different Seasons, Journal of Applied Animal Research, 23:2, 185-194, DOI: 10.1080/09712119.2003. 9706420.

Al-Hamed, A.M.Y (2010).The Impact Of Bird Density And The Number Of Fodder And Different Temperatures And The Interaction Between Them On Some Productive And Physiological Characteristics. College of Agriculture and Forestry / University of Mosul.

Al-Naimi, A.M. Th. Q. (2018). Effect Of Adding Green Tea Powder (Camellia Sinensis) In The Diet On Productive Performance And Microbial Content Of Quail Birds Raised Under High Temperature. Master Thesis. College of Agriculture and Forestry / University of Mosul. 
Mesopotamia J. of Agric.

Vol. (48) No. (1) 2020
ISSN: $2224-9796$ (Online)

ISSN: 1815 - 316 X (Print)
المجلد (48) العدة زردة (1) الــر افديـن 2020

Al-Sheikhli, F. I. Abdul- Jabbar (2003). Poultry Diseases. Atlas Printing Co. Ltd. Baghdad, Iraq.

Anonymous (1994). National Research Council (N.R.C.) Nutrient Requirements Of Poultry. National Academy Of Science, Washington, D.C.

Anonymous (2003).(S.A.S). Statistical Analysis System User's Guide (Version 9.1.3). SAS Institute Inc., Cary North Carolina, U.S.A.

Belewn, M.A ; O.A. Olatunde and T.A. Giwa (2009). Under unitized medicinal plants and spices chemical composition and phytochemical properties. Journal of Medical Plants Research. 3(12):1099-1103.

Çabuk, M. ; A. Alcicek ; M. Bozkurt and N. Imre (2003). Antimicrobial properties of the essential oils isolated from aromatic plants and using possibility as alternative feed additives. $11^{\text {th }}$ National Animal Nutrition Congress. Pp;184-187.

Crespo, N. and E. Esteve-Garcia.(2002). Nutrient and fatty acid deposition in broilers fed different fatty acid profiles. Poultry Science, 81: 1533-1542.

Dipti, P. ; B. Yogesh ; A.K. Kain ; T. Pauline ; B. Anju ; M. Sairam ; B. Singh ; S.S. Mongia ; G.I. Kumar and W. Selvamurthy (2003). Lead induced oxidative stress beneficial effects of Kampuchea tea. Biomedical And Environmental Sciences: BES, , 16(3): 276-282.

Duncan, D.B. (1955). Multiple Range And Multiple F Test.

El-Deek, A.A ; M.A. Al-Harthi; M. Osman ; F. Al-Jassas and R. Nassar (2011). Effect of different level of green tea (camellia sinensis) as a substitute for oxtetracycline as a growth promoter in broilers diets containing two crude protein levels. Archiv für Geflügelkunde. 76 (2):88 -98.

Feddes, J.J. ; E.J. Emmanuel and M.J. Zuidhof (2002). Broiler performance, body weight variance, feed and water intake, and carcass quality at different stocking densities. Poultry Science, 81:774-779.

Friedewald, W. ; Y. Levy and N. Fredrickson (1972). Estimation of the concentration of low density lipoprotein cholesterol in plasma without use of preparative ultra-centrifuge. Clinical Chemistry, $18: 499-502$.

Giannenas, I. ; P. Florou-Paneri ; M. Papazahariadou ; E. Christaki ; N.A.Botsoglou and A.B. Spais (2003): Effect of dietary supplementation with oregano essential oil on performance of broilers after experimental infection with Eimeria tenella, Archives of Animal Nutrition, 57:2, 99-106.

Hassan, A.M.(2009). Evaluation Of Productive Performance Of Two Broiler Chicks Bred Under Different Levels Of Density. Master Thesis -College of Agriculture - University of Duhok.

Hong, Y. ; W. Bin and H. Yiyu cheng (2009). High throughput chemiluninescence platform for evaluating anti-oxidative activity of total flavonoid glycosides from plant extracts. Food Chemistry., 115: 380-386.

Houshmand, M. ; K. Azhar ; I. Zulkifli ; M.H. Bejoand K. Amyab (2012).Effect of prebiotic, protein level, and stocking on performance, immunity and stress indicators broiler. Poultry Science, 91:393-401. 
Mesopotamia J. of Agric. Vol. (48) No. (1) 2020
ISSN: $2224-9796$ (Online)

ISSN: 1815 - 316 X (Print)
المجلد (48) العدة زراعـة الــر افديـن 2020

Jiang, F.; Y. Guo ; D. Salvimini and G.J. Dusting (2003). Superoxide dismutase mimetic M40403 improves endothelial function in Apo lipoprotein (E)deficient mice. British Journal of Pharmacology. 139(6):1127-1134.

Latshaw, J.D.(2008). Daily energy intake of broiler chickens is altered by proximate nutrient content and form of the diet. Poultry Science, 87: 89-95.

Metheringham, J. and Dr. Hubrecht (1996). Poultry in transit A cause for concern?. British Veterinary Journal, 152: 247-249.

Rice, E.C.A. ; N.J. Miller; P.G Bolwell ; P.M. Bramley and J.B. Pridham (2001). The relative antioxidant activities of plant-derived polyphenol flavonoids. Free Radical Research., 22: 375-383.

Saeed, S. and P. Taria (2006).Effect of some seasonal vegetables and fruits on growth bacteria. Pakistan Journal of Biological Sciences $q$ (8): 1547-1551.

Sahin, K ; C. Orhan ; M. Tuzcu ; S. Ali ; N. Sahin and A. Hayirli (2010). Epigallocatechin-3-gal late prevents lipid peroxidation and enhances antioxidant defense system via modulating hepatic nuclear transcription factors in heat-stressed quails. Poultry Science, 89:2251-2258.

Siegel, P.B. and C.F. Honaker (2014). General principles of stress and well-being. In Livestock Handling and Transport: Fourth Edition (Pp. 14-22). CABI International. https://doi.org/10.1079/9781780643212.0014

Steel, R.G.D. and Torrie, J.H., (1960). Principles and procedures of statistics with special reference to the biological sciences. New York, Mcgraw hill.

Suad, M. Aou-abdallah (2010). Study Of Some Biological Effects Of Green Tea Plant Extract On Antioxidant And Antibacterial Activity. Master Thesis, Department of Animal Biology, Faculty of Natural and Life Sciences, University of Montour Constantine.

Tabler, G.T. ; I.L. Barrey, and A.M. Mendenhall (2004). Mortality patterns associated with commercial broiler production. Avian advice Journals, 6(1).

Tietz, N.W. (1995). Clinical Guide To Laboratory Tests . $3^{\text {rd }}$ Ed.Philadelphia.PA : WB. Saunders Company.268 - 273 . 